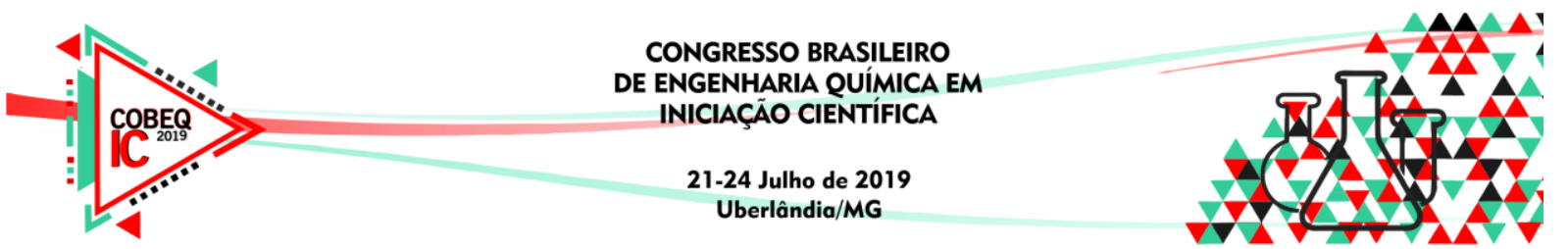

\title{
SECAGEM POR ATOMIZAÇÃO DA FOLHA DE MARACUJÁ- AMARELO (Passiflora edulis Sims)
}

\author{
P. S. CAMPOS, M. E. OLIVEIRA, C. I. SILVA, G. C. R. Silva e T. P. V. B. DIAS \\ Universidade Federal de São João Del Rei, Laboratório de Engenharia de Processos e \\ Tecnologia \\ priscilla.silvacampos@yahoo.com.br
}

RESUMO - No Brasil, as espécies do gênero Passiflora L, denominadas popularmente por "maracujá", são utilizadas como ansiolítico, sedativo, diurético e analgésico e juntamente com as suas folhas, que também são utilizadas na medicina no tratamento de ansiedade e nevralgia. Além disso, as folhas também apresentam propriedades ansiolíticas. Processos de secagem por atomização são uma excelente alternativa para a secagem de alimentos com alto valor agregado, pois, através da microencapsulação, preservam as características sensoriais, nutricionais e vida útil do produto. O presente trabalho avaliou a influência da temperatura (111 a $170^{\circ} \mathrm{C}$ ) e concentração de adjuvante (Aerosil, 21,44 a 51,55\%) na microencapsulação por Spray dryer dos compostos bioativos das folhas da espécie Passiflora edulis Sims comumente conhecida como maracujá amarelo ou maracujá azedo, buscando uma maior preservação desses compostos. Logo, após a secagem por atomização as partículas encapsuladas foram caracterizadas quanto à higroscopicidade, solubilidade, teor de compostos redutores, carotenoides, flavonoides e rendimento da secagem. No pó atomizado das folhas de maracujá azedo obtivemos o maior rendimento no valor de $52,67 \%$, que ocorreu utilizando uma temperatura de $141^{\circ} \mathrm{C}$ e concentração de adjuvante de $36 \%$, com solubilidade de $54,76 \%$, higroscopicidade de 22,28\%, flavonoides $11,46 \mathrm{mg}$ QU/g, carotenoides 0,0873 $\mathrm{mg} / 100 \mathrm{~g}$ e compostos redutores 59,88 mg GAE/g pó.

\section{INTRODUÇÃO}

A técnica de secagem por nebulização pode ser uma excelente alternativa para secagem de alimentos com alto valor agregado, pois além de aumentar a sua vida de prateleira pode vir, também, a preservar seus compostos biologicamente ativos, bem como o flavour.

$\mathrm{O}$ método de secagem por atomização gera produtos de alta qualidade, devido às elevadas temperaturas associadas ao curto tempo de residência do produto na câmera de secagem que resultam na elevada taxa de evaporação, dentre as suas vantagens podemos citar, controle da uniformidade da granulometria do produto, processo rápido e contínuo, maior estabilidade físico-química do produto obtido, aplicabilidade para produtos termo sensíveis e baixos custos de processo conforme Leimann et al., (2009); Oliveira \& Petrovick, (2010).

As condições de operações usadas na secagem e processamento térmico de bioprodutos podem ter considerável impacto nas propriedades e custo do produto, gerando diferentes graus de perda de compostos ativos. Assim, este projeto, tem por objetivo o estudo 


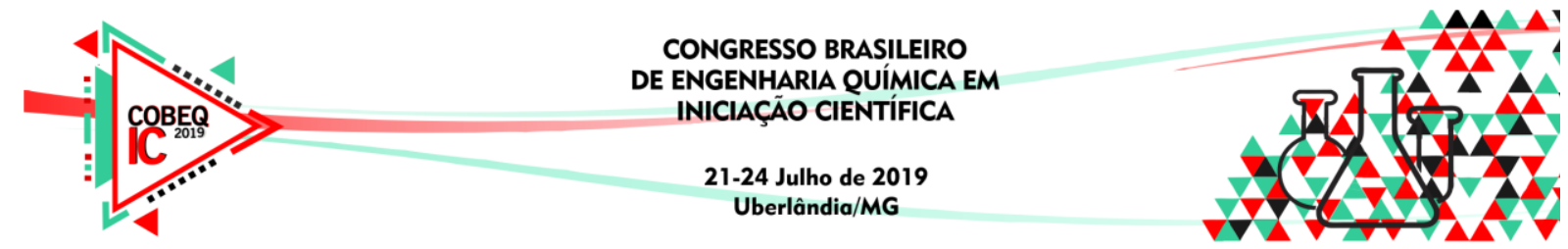

dos efeitos das variáveis de secagem por Spray Dryer (temperatura e concentração de adjuvante) na folha de maracujá azedo.

\section{SECAGEM POR ATOMIZAÇÃO (SPRAY DRYER)}

Para otimização do processo de secagem variou-se os seguintes parâmetros: temperatura e concentração de Aerosil, conforme planejamento experimental baseado na Metodologia de Superfície de Resposta. Foi realizado um delineamento composto central rotacional (DCCR), sendo um fatorial completo 22, incluindo 3 pontos axiais e 3 repetições no ponto central, totalizando 11 ensaios.

\section{CARACTERIZAÇÃO DO PÓ ATOMIZADO}

Os pós atomizados em Spray dryer foram caracterizados com relação à solubilidade, higroscopicidade, carotenoides, flavonoides e de teor de compostos redutores conforme Granato et. al. (2008) descrito a seguir. Sendo que, todas as análises realizadas em triplicata. $\mathrm{O}$ índice de solubilidade em água foi determinado conforme o método descrito por CanoChauca e et. al (2005).

Para o cálculo da higroscopicidade e rendimento da secagem foi necessário à análise do teor de água, que foi determinada conforme AOAC (2002).

A higroscopicidade foi determinada de acordo com o método proposto por Tonon et. al. (2009). A determinação dos carotenoides foi realizada segundo o método proposto por Higby (1962).

A determinação de flavonoides foi realizada segundo o método proposto pela ANVISA (2010), Para a quantificação do teor de flavonoides foi realizada uma curva padrão de concentração de quercetina $(\mathrm{g})$ por absorbância $(\mathrm{nm})$.

O conteúdo de compostos redutores totais foi determinado pelo método de Folin Ciocalteu, segundo metodologia descrita por Singleton et. al. (1999). Para a quantificação do teor de compostos redutores foi realizada uma curva padrão de concentração de ácido gálico (g) por absorbância (nm). Os resultados foram expressos em mg GAE / g pó.

O rendimento dos processos de secagem foi calculado conforme a Equação 01.

Rendimento $(\%)=\frac{P_{P A}}{\left(P_{S T}\right)+\left(P_{A}\right)} x 100$

onde, $P_{P A}$ corresponde ao peso do pó atomizado, $P_{S T}$ representa o peso dos sólidos totais presentes no extrato aquoso e $P_{A}$, o peso de adjuvante que foi utilizado na secagem. 


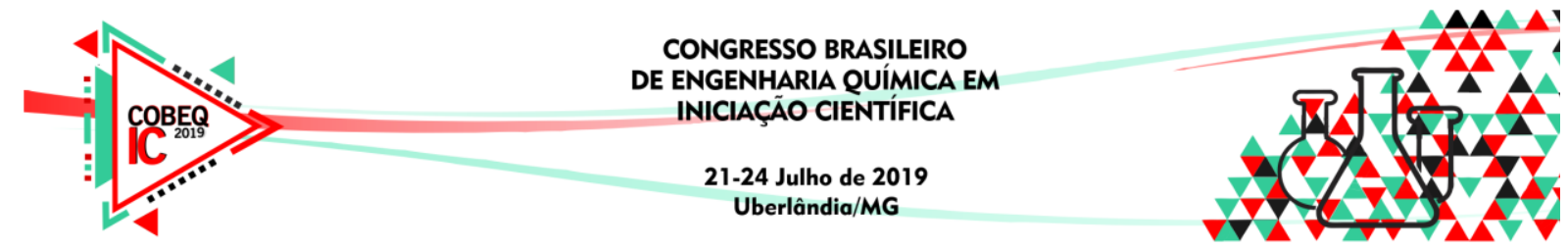

\section{RESULTADOS E DISCUSSÃO}

Na Tabela 1 são apresentados os dados obtidos para a caracterização dos pós atomizados obtidos na secagem dos extratos de folhas de maracujá azedo.

Tabela 1 - Caracterização dos pós de maracujá azedo (Passiflora edulis Sims) e rendimento de secagem.

\begin{tabular}{ccccccc}
\hline Ensaio & $\begin{array}{c}\text { Compostos Fenólicos } \\
\text { (mg GAE/g pó) }\end{array}$ & $\begin{array}{c}\text { Carotenoides } \\
(\mathbf{m g} / \mathbf{1 0 0 g})\end{array}$ & $\begin{array}{c}\text { Flavonoides } \\
(\mathbf{m g Q U} / \mathbf{g})\end{array}$ & $\begin{array}{c}\text { Rendimento } \\
(\mathbf{\%})\end{array}$ & $\begin{array}{c}\text { Higroscopicidade } \\
(\boldsymbol{\%})\end{array}$ & $\begin{array}{c}\text { Solubilidade } \\
(\mathbf{\%})\end{array}$ \\
\hline $\mathbf{1}$ & 41,91 & 16,075 & 13,66 & 43,32 & 22,02 & 61,96 \\
$\mathbf{2}$ & 57,33 & 7,909 & 14,11 & 50,14 & 21,25 & 53,96 \\
$\mathbf{3}$ & 55,11 & 16,654 & 15,79 & 33,49 & 19,98 & 53,78 \\
$\mathbf{4}$ & 63,99 & 1,929 & 13,16 & 41,35 & 19,95 & 55,82 \\
$\mathbf{5}$ & 62,77 & 4,630 & 12,59 & 33,01 & 21,21 & 58,31 \\
$\mathbf{6}$ & 69,20 & 4,244 & 13,09 & 48,63 & 21,13 & 57,60 \\
$\mathbf{7}$ & 67,65 & 5,337 & 9,41 & 40,62 & 23,01 & 63,20 \\
$\mathbf{8}$ & 53,33 & 3,858 & 11,87 & 34,49 & 19,48 & 52,27 \\
$\mathbf{9}$ & 47,90 & 7,009 & 11,17 & 43,16 & 21,91 & 56,71 \\
$\mathbf{1 0}$ & 62,54 & 7,845 & 11,74 & 41,34 & 21,67 & 55,56 \\
$\mathbf{1 1}$ & 59,88 & 8,423 & 11,46 & 42,25 & 22,28 & 54,76 \\
\hline
\end{tabular}

O maior rendimento obtido foi de $43,167 \%$, este valor é satisfatório, visto que, atomizadores laboratoriais apresentam baixo rendimento, alguns raramente fornecem rendimentos acima de 50\%, mesmo quando se utiliza grande quantidade de agentes encapsulantes conforme Labmaq (2014).

A solubilidade do pó em água variou de 52,27 a $63,20 \%$, no caso em estudo é desejável uma maior solubilidade, uma vez que, o produto obtido pode ser utilizado na formulação de diversos produtos alimentícios, para enriquecimento nutricional.

Observa-se que os pós obtiveram valores relativamente baixos de higroscopicidade, 19,48 a 23,01\%. A baixa higroscopicidade do pó é fundamental e desejável, pois com a absorção de água as micropartículas podem mudar sua estrutura física, aderência e formar aglomerados, perdendo, assim, sua estrutura e função, além de tornarem-se susceptíveis a proliferação de microrganismos de acordo com o trabalho de Porras-Saavedra et al., (2015).

Observa-se ainda que os teores de compostos redutores, carotenoides e flavonoides presentes nas folhas de maracujá azedo, foram de 41,91 a 69,20 mg GAE/g pó; 0,0200 a $0,1727 \mathrm{mg} / 100 \mathrm{~g}$ e 9,41 a $15,79 \mathrm{mgQU} / \mathrm{g}$, respectivamente.

Para análise da significância das variáveis independentes dentro das respostas, os resultados obtidos foram submetidos à análise estatística. Os coeficientes de determinação, regressão e seus respectivos p-valores são apresentados na Tabela 2. Para a análise estatística considerou-se um nível de significância de $5 \%$ (p-valor $<0,05)$. 


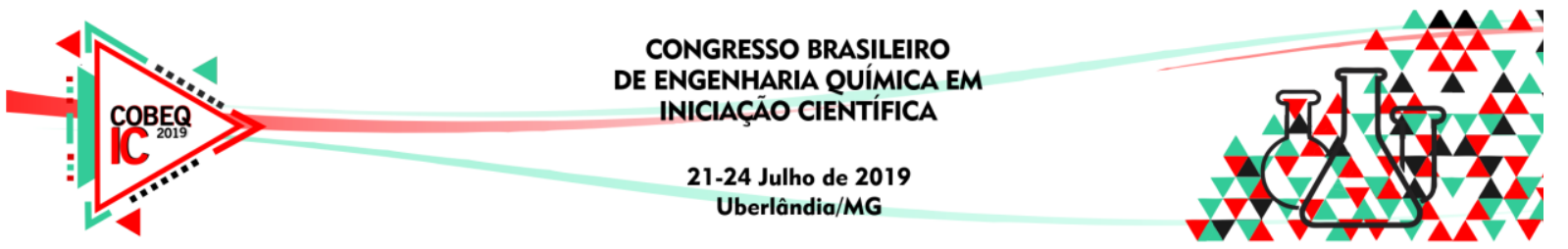

Tabela 2 - Estimativa do p-valor e coeficiente de regressão (C.R.) para rendimento, higroscopicidade, solubilidade, carotenoides, teor de compostos fenólicos e flavonoides do pó atomizado da folha de maracujá azedo (Passiflora edulis Sims).

\begin{tabular}{cccccccccccccccc}
\hline & \multicolumn{1}{c}{ Rendimento } & \multicolumn{1}{c}{ Higroscopicidade } & \multicolumn{2}{c}{ Solubilidade } & Carotenoides & Compostos Fenólicos & Flavonoides \\
\hline & $\mathrm{R}^{2}$ & 0,8812 & $\mathrm{R}^{2}$ & 0,9220 & $\mathrm{R}^{2}$ & 0,6575 & $\mathrm{R}^{2}$ & 0,3694 & $\mathrm{R}^{2}$ & 0,3335 & $\mathrm{R}^{2}$ & 0,5234 \\
& $\mathrm{CR}$ & $\mathrm{p}$-valor & $\mathrm{CR}$ & $\mathrm{p}$-valor & $\mathrm{CR}$ & $\mathrm{p}$-valor & $\mathrm{CR}$ & $\mathrm{p}$-valor & $\mathrm{CR}$ & $\mathrm{p}$-valor & $\mathrm{CR}$ & $\mathrm{p}$-valor \\
\hline $\mathrm{T}(\mathrm{L})$ & 4,5963 & $0,0051^{*}$ & $-0,1141$ & 0,4858 & $-0,8705$ & 0,2631 & $-3,0742$ & 0,1732 & 4,1742 & $0,0125^{*}$ & $-0,1841$ & 0,7664 \\
$\mathrm{~T}(\mathrm{Q})$ & 0,0069 & 0,9955 & $-0,4935$ & $0,0412^{*}$ & 0,7479 & 0,4046 & $-0,2630$ & 0,9136 & 0,2244 & 0,7273 & 1,3017 & 0,1214 \\
$\mathrm{~A}(\mathrm{~L})$ & $-3,4111$ & $0,0169^{*}$ & $-1,0415$ & $0,0010^{*}$ & $-2,6515$ & $0,0121^{*}$ & $-0,5816$ & 0,7760 & $-0,0489$ & 0,9266 & 0,5824 & 0,3665 \\
$\mathrm{~A}(\mathrm{Q})$ & $-1,6256$ & 0,2178 & $-0,4560$ & 0,0529 & 0,7379 & 0,4104 & $-0,6248$ & 0,7971 & $-2,5231$ & $0,0458^{*}$ & 0,2017 & 0,7844 \\
$\mathrm{~T} \mathrm{x} \mathrm{A}$ & 0,2600 & 0,8570 & 0,1850 & 0,4281 & 2,5100 & 0,0500 & $-1,3505$ & 0,6427 & $-1,6350$ & 0,1332 & $-0,7700$ & 0,3960 \\
\hline
\end{tabular}

Onde T é a temperatura em ${ }^{\circ} \mathrm{C}$, A é a \% de adjuvante adicionada, L indica o termo linear e Q o termo quadrático. (*) indicam variáveis estatisticamente significativas, a $5 \%$ de significância.

As variáveis dependentes, rendimento e higroscopicidade apresentaram elevados coeficientes de determinação, 0,8812 e 0,9220 respectivamente, indicando uma confiabilidade da análise estatística. A solubilidade apresentou um valor de coeficiente de determinação de 0,6575, valor este aceitável já que o processo de secagem piloto em Spray dryer que envolve vários problemas operacionais já descritos pelo trabalho de Toneli et al. (2010). Já carotenoides, compostos redutores e flavonoides apresentaram menor coeficiente de determinação, 0,$337 ; 0,369 ; 0,333$ e 0,523 valores baixos indicando que o modelo não se ajustou aos dados obtidos. Observou-se ainda que a temperatura fosse significativa para rendimento, higroscopicidade e compostos fenólicos, já a concentração de Aerosil foi significativa para rendimento, solubilidade e compostos redutores.

Analisando o coeficiente de regressão, observou-se que sendo que quanto maior a temperatura e menor a concentração de Aerosil, maior o rendimento obtido. O efeito da temperatura no rendimento do processo de secagem pode ser justificado pelo fato das temperaturas mais elevadas facilitarem o processo de secagem, através da redução da tensão superficial e viscosidade, o que facilita a formação de gotículas, gerando maiores rendimentos de acordo com Oliveira \& Petrovick (2010).

Já para a higroscopicidade o efeito da temperatura foi o termo quadrático, indicando que em temperaturas intermediárias as amostras apresentam maior higroscopicidade. Para a solubilidade somente a concentração de Aerosil foi significativa, indicando que menores concentrações de Aerosil aumentam a solubilidade. O Aerosil é um adjuvante altamente hidrofóbico, afetando assim a solubilidade do produto conforme Araújo et al. (2010), ou seja, elevadas quantidades de Aerosil levam a menores solubilidades. Por ser hidrofóbico, o Aerosil também pode estar intimamente ligado a higroscopicidade do produto, dificultando que este retenha água, levando a um produto menos higroscópico. Embora o coeficiente de determinação tenha sido baixo para o teor de compostos redutores, o modelo nos diz que maiores temperaturas e concentrações intermediárias de Aerosil, levam a amostra com maior teor de compostos redutores.

A temperatura e a concentração de adjuvantes não demonstrou significância para as análises de carotenoides e flavonoides, isto pode ser devido à temperatura de entrada muito elevada, podendo quebrar o equilíbrio entre taxa de evaporação da água e formação do filme, 


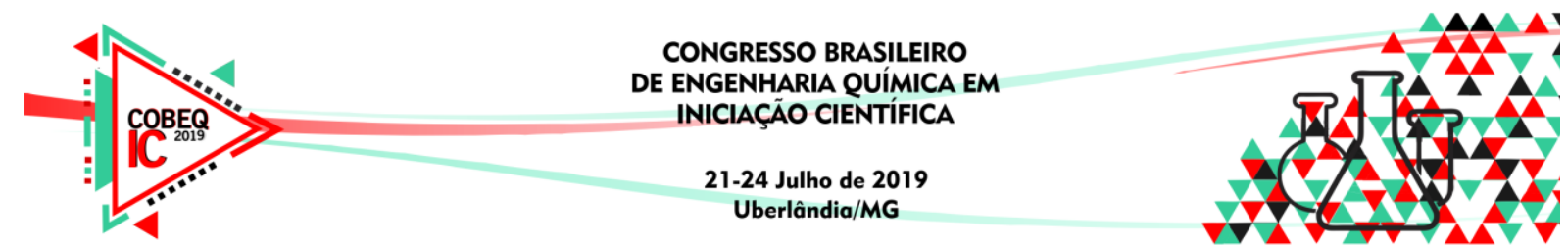

levando a quebra da microcápsula como demonstrado por Shu et al. (2006), podendo deduzir que há uma baixa retenção de compostos como carotenoides e flavonoides quando a maioria das microcápsulas foram quebradas. Em nenhuma das análises, a interação direta entre temperatura e concentração do adjuvante Aerosil foi significativa.

A partir dos resultados experimentais, analisou-se o ajuste das regressões por meio do teste F conforme descrito na Tabela 3.

Tabela 3 - Valores de F-cal e p-valor da regressão das respostas de rendimento e higroscopicidade.

\begin{tabular}{cccc}
\hline & Rendimento $(\%)$ & Higroscopicidade $(\%)$ & Solubilidade $(\%)$ \\
\hline F-cal & 2,7874 & 0,1089 & 0,9232 \\
p-valor & 0,1425 & 0,9853 & 0,5339 \\
\hline
\end{tabular}

Considerando um nível de significância de 5\%.

O rendimento, a higroscopicidade e a solubilidade não obtiveram, estatisticamente, um bom ajuste da regressão, pois os valores de $F_{\text {calculado }}$ foram menores que o $F_{\text {tabelado }}(5,05)$, não sendo possível a construção das superfícies de resposta e contorno.

\section{CONSIDERAÇÕES FINAIS}

Através deste estudo, pode-se concluir que para o pó atomizado das folhas de maracujá azedo, quanto maior a temperatura e menor a concentração de Aerosil, maior o rendimento obtido, sendo que em temperaturas intermediárias apresentam maior higroscopicidade. Já para a solubilidade as análises mostraram que menores concentrações de Aerosil aumentam a solubilidade. Embora o coeficiente de determinação tenha sido baixo para o teor de compostos redutores, o modelo nos diz que maiores temperaturas e concentrações intermediárias de Aerosil, levam a uma amostra com maior teor de compostos redutores.

Para as folhas de maracujá azedo o propósito geral deste trabalho, que era estabelecer as melhores condições de secagem por atomização em Spray dryer não foi atendido, visto que modelo não se ajustou significativamente aos dados obtidos.

\section{REFERÊNCIAS BIBLIOGRÁFICAS}

ANVISA; Farmacopeia Brasileira. v2. 5ª ed. Brasília. 2010

ARAÚJO, R. R.; TEIXEIRA, C. C. C.; FREITAS, L. A. P. The preparation of ternary solid dispersions of an herbal drug via spray drying of liquid feed. Drying Technology, 2010.

AOAC - ASSOCIATION OF OFFICIAL ANALYTICAL CHEMISTS. Official methods of analysis of Association of official Analytical Chemists International. $18^{\text {th }}$ ed. Gainsthersburg: Horwitz, 2002 


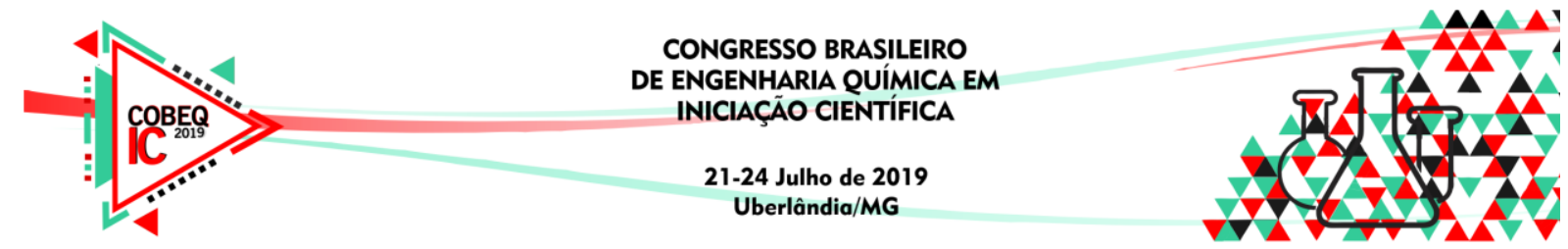

CANO-CHAUCA, M.; et al. Effect of the carriers on the microstructure of mango powder obtained by spray drying and its functional characterization. Innovative Food Science \& Emerging Technologies, v. 6, n. 4, p. 420-428, 2005.

GRANATO, D.; et. al. Antioxidant activity, total phenolics and flavonoids contents: Should we ban in vitro screening methods?. Food Chemistry,v. 264, n. 6, p. 471-475, 2018.

JINAPONG, N.; SUPHANTHARIKA, M.; JAMNONG, P. Production of instant soymilk powders by ultrafiltration, spray drying and fluidized bed agglomeration. Journal of Food Engineering, v. 84, n. 2, p. 194-205, 2008.

HIGBY, W. K; A simplified method for determination of the carotenoid distribution in natural and carotene fortifield Orange Juice. Journal of food Science, v. 27, p.42-49. 1962.

LABMAQ. Manual de Operação - Secador por nebulização mini "Spray dryer" Modelo MSDi 1.0. LABMAQ DO BRASIL LTDA, 2014.

LEIMANN, F.V. et al.. Antimicrobial activity of microencapsulated lemongrass essential oil and the effect of experimental parameters on microcapsules siz and morphology. Materials Science and Engineering, v. 29, 430-436, 2009.

PORRAS-SAAVEDRA, J.; PALACIOS-GONZÁLEZ, E.; LARTUNDO-ROJAS, L. et al. Microstructural properties and distribution of components in microparticles obtained by spray-drying. Journal of Food Engineering, v. 152, p. 105-112, 2015.

OLIVEIRA, O. W.; PETROVICK, P. R. Secagem por aspersão (spray drying) de extratos vegetais: bases e aplicações. Rev Bras Farmacogn, v. 20, n. 4, p. 641-50, 2010.

SINGLETON, V. L.; ORTHOFER, R.; LAMUELA-RAVENTÓS, R. M. Analysis of total phenols and other oxidation substrates and antioxidants by means of folin-ciocalteu reagent. Methods in enzymology, n. 299, p. 152-178, 1999.

TONELI, J.; PARK, K.; NEGREIROS, A. et al. Spray-drying process optimization of chicory root inulin. Drying Technology, v. 28, n. 3, p. 369-379, 2010.

TONON, R. V.; BRABET, C.; PALLET, D. et al. Physicochemical and morphological characterisation of açai (Euterpe oleraceae Mart.) powder produced with different carrier agents. International journal of food science \& technology, 2009. 